\title{
Commentary Conditional mouse models demonstrate oncogene-dependent differences in tumor maintenance and recurrence
}

\author{
Maddalena T Tilli and Priscilla A Furth \\ Correspondence: Priscilla A Furth (e-mail: paf3@georgetown.edu) \\ Published: 30 May 2003 \\ Breast Cancer Res 2003, 5:202-205 (DOI 10.1186/bcr614) \\ (C) 2003 BioMed Central Ltd (Print ISSN 1465-5411; Online ISSN 1465-542X)
}

Division of Human Genetics, University of Maryland, Baltimore, and Lombardi Cancer Center, Georgetown University, Washington, DC, USA

\begin{abstract}
Diversity in the pathophysiology of breast cancer frustrates therapeutic progress. We need to understand how mechanisms activated by specific combinations of oncogenes, tumor suppressors, and hormonal signaling pathways govern response to therapy and prognosis. A recent series of investigations conducted by Chodosh and colleagues offers new insights into the similarities and differences between specific oncogenic pathways. Expression of three oncogenes relevant to pathways activated in human breast cancers (c-myc, activated neu and Wnt1) were targeted to murine mammary epithelial cells using the same transgenic tetracycline-responsive conditional gene expression system. While the individual transgenic lines demonstrate similarly high rates of tumor penetrance, rates of oncogene-independent tumor maintenance and recurrence following initial regression are significantly different, and are modifiable by mutations in specific cooperating oncogenes or loss of tumor suppressor gene expression. The experiments make three notable contributions. First, they illustrate that rates of tumor regression and recurrence following initial regression are dependent upon the pathways activated by the initiating oncogene. The experiments also demonstrate that altered expression or mutation of specific cooperating oncogenes or tumor suppressor genes results in different rates of tumor regression and recurrence. Finally, they exemplify the power of conditional mouse models for elucidating how specific molecular mechanisms give rise to the complexity of human cancer.
\end{abstract}

Keywords: conditional transgenic mouse models, oncogene dependence, reversible tumorigenesis, tumor maintenance, tumor regression

\section{Introduction}

Cancer is a multistage process. Cells are selected after accumulating successive genetic lesions in oncogenes and tumor suppressor genes, resulting in independence from the normal restraints that regulate growth, proliferation, survival, differentiation and migration [1-5]. Conditional transgenic mouse models expressing oncogenes involved in human cancer pathways have revolutionized the way in which we define the contributions that these oncogenes lend to the process of tumorigenesis. Adaptation of the tetracycline-responsive gene expression system to transgenic mice [6-9] prepared the way for development of conditional models in which precise timing of oncogene exposure in specific tissues initiated events that model those that occur during the stepwise progression of human oncogenesis [10-18].

A first application of these models was to study the potential for reversal of a dysplastic or malignant phenotype when expression of an initiating oncogene was downregulated. Determining the effect of loss of expression of the initiating oncogene establishes the requirement of the initiating oncogene for maintenance of preneoplasia and/or neoplasia. Experiments to date reveal provocative differences in preneoplastic or neoplastic reversal between different initiating oncogenes and target tissues. Reversibility 
of salivary oncogenesis initiated by expression of the complex oncoprotein Large Simian Virus 40 T antigen, which disrupts both pRB-related and p53 tumor suppressor pathways, is interrupted at a preneoplastic stage in a time-dependent manner [11]. In contrast, melanomas initiated by $H$-Ras [12], lung carcinomas initiated by $K$-Ras [13], and leukemia initiated by $B C R-A B L$ [14] remain reversible through the neoplastic stage after expression of the initiating oncogene is downregulated. Reversibility in different tissues following downregulation of the single initiating oncogene c-myc can be compared in mammary adenocarcinomas [15] and in lymphomas [16]. Whereas $90 \%$ of the lymphomas regress after c-myc abrogation, regression of mammary adenocarcinomas is limited by ras mutation. Seventy-eight percent of mammary adenocarcinomas without ras mutation regress, while adenocarcinomas with ras mutation do not regress after c-myc downregulation.

In these experiments neoplastic reversal is due either to rapid apoptosis of tumor cells [12-14,16] or to redifferentiation of cells causing arrest of proliferation and loss of malignant potential [16]. These models are important since they suggest that ablation of signaling from one oncogenic pathway may be sufficient to reverse a malignant phenotype. In some models it is possible that tumor cells expressing an oncogene may have undergone adaptive transcriptional changes that lead to their dependence on continued expression of the oncogene for growth and survival. These adaptive changes might not allow the tumor cells to switch to other proliferative pathways swiftly enough to prevent activation of apoptotic machinery [13].

Oncogene-independent recurrent growth of tumors in animals with regressed primary tumors was reported in a small percentage of cases in the H-Ras-induced melanoma [12], in c-myc-induced lymphomas [16] and in c-myc-induced mammary adenocarcinomas [15]. Additionally, in a line of mice with longer leukemia latency, all mice in remission due to downregulation of the $B C R-A B L$ transgene developed a $B C R$ - $A B L$-independent, rapidly progressing, B-cell leukemia [14]. It was hypothesized that the prolonged exposure of the cells to $B C R-A B L$ allowed for the development of secondary mutations that bypass reliance on the initial transforming event. In the c-myc mammary adenocarcinoma model, the ras mutation was identified as a genetic event that promotes c-mycindependent tumor growth [15].

\section{Reversibility and recurrence of mammary adenocarcinomas and metastases induced by activated Neu}

Chodosh and colleagues have extended and amplified these studies by examining the reversibility and oncogeneindependent recurrence of different initiating oncogenes in the same target tissue, the mammary gland (Fig. 1).
Figure 1

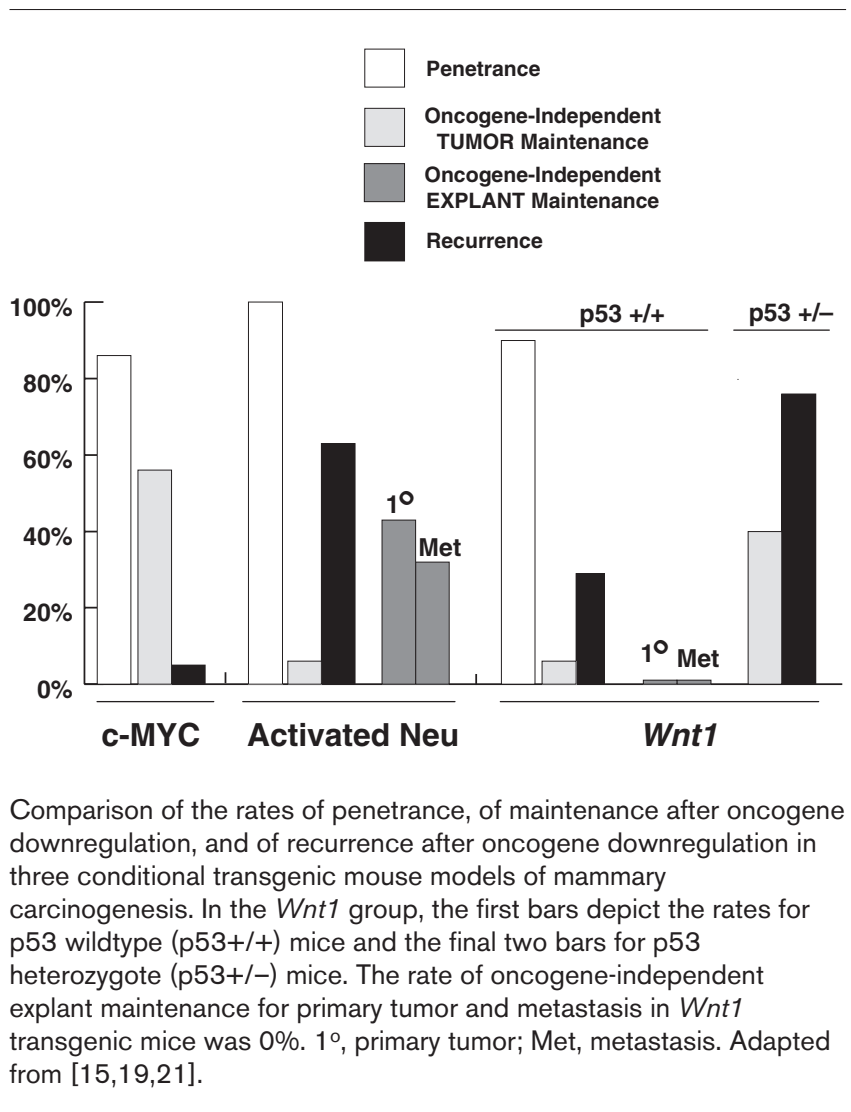

Transgenic mice with conditional expression of activated Neu in mammary epithelial cells were designed (MTB/TAN mice) to examine the dependence of continued oncogene expression on activated Neu-initiated tumors and metastases [19]. Expression of activated Neu causes hyperplastic abnormalities in the mammary ductal tree, in the ductal hyperplasia, and in the invasive mammary nodular carcinomas in $100 \%$ of the mice. Downregulation of activated Neu in double transgenic MTB/TAN female mice with mammary carcinomas showed that $94 \%$ of tumors are reversible and regress to a nonpalpable state. Even metastatic lesions are dependent on activated Neu for growth and also regress following abrogation of transgene expression. However, as frequently occurs in human cancers following initial therapeutic success, a significant percentage $(63 \%)$ of the regressed cancers eventually recur.

An intriguing feature of the experiments was the fact that the percentages of primary tumors and metastases demonstrating oncogene-independent maintenance was increased when they were first transplanted into syngeneic hosts before abrogation of transgene expression. Stromal cells surrounding tumors can secrete growth and survival factors (such as matrix metalloproteinases), which may promote cancer development, cancer progression, and cancer metastasis by altering the tumor environment 
[20]. For example, matrix metalloproteinases may act in transformation by inducing apoptosis in anchorage-dependent cells and/or by modifying cell cycle checkpoints through alterations in cell adhesion. The increase in primary tumors and metastases that resulted after transplantation illustrates that tumor regression did not necessarily occur after loss of oncogene expression. The increase raises the possibility that this model can be utilized to explore how the tumor environment can modify cancer cell behavior and how stromal changes are linked to primary epithelial cell oncogenic events.

\section{Loss of one p53 allele alters reversal and recurrence of Wnt-induced mammary adenocarcinomas}

Chodosh and colleagues used the same conditional system to examine the requirement of continued Wnt1 expression in the maintenance and recurrence of mammary cancers by developing double transgenic mice with conditional expression of Wnt1 (MTB/TWNT mice) [21]. In MTB/TWNT double transgenic females, induction of Wnt 1 expression by doxycycline administration results in increased ductal side branching and in precocious lobuloalveolar development. Upon chronic Wnt1 exposure, ductal hyperplasia develops into invasive mammary adenocarcinomas that metastasize to the lung. Similar to activated $\mathrm{Neu}$, abrogation of $\mathrm{Wnt}$ signaling by doxycycline withdrawal leads to rapid regression of mammary adenocarcinomas and lung metastases in $94 \%$ of the mice. But intriguingly, rates of tumor reoccurrence and the requirement for continued oncogene expression for tumor maintenance following transplantation were significantly different from those in the activated Neu model (Fig.1). Tumors recur in only 29\% of the regressed Wnt 1 tumors, and all of the transplanted tumors and metastases regressed after loss of oncogene expression.

As the next step, Chodosh and colleagues examined the role of the specific tumor suppressor gene p53 in oncogene-independent tumor maintenance and recurrence. Even though p53 is not required for regression of Wnt1induced tumors, $40 \%$ of the tumors that develop in p53 heterozygous mice do not regress when Wnt1 is downregulated, and a high percentage (76\%) of the adenocarcinomas that do regress subsequently recur.

\section{Conclusion}

Conditional transgenic mouse models allow investigators to model possible therapeutic consequences of oncogene downregulation on the maintenance of malignancy and recurrence. Oncogenic pathways can be examined in individual tissues and cancer systems to determine whether there will be oncogene-independent growth, and if so to which alternative pathways the cells turn in order to bypass oncogene dependence. Results from these experi-
Experiments by Chodosh and colleagues illustrate the power of conditional transgenic mouse systems to investigate the impact of different initiating oncogenes on mammary oncogenesis, on oncogene-independent tumor maintenance, and on recurrence following initial regression. The results exemplify the differences between oncogenes. While $56 \%$ of c-myc-induced mammary cancers demonstrate oncogene-independent tumor maintenance, only 6\% of activated Neu-induced and Wnt1-induced mammary cancers demonstrate oncogene-independent tumor maintenance. While only a fraction of the c-mycinduced mammary cancers that regress after downregulation of oncogene expression subsequently recur, $63 \%$ of regressed activated Neu mammary cancers and 29\% of regressed Wnt1 mammary cancers recur. The Chodosh laboratory also shows us examples of how mutations in cooperating oncogenes, changes in tumor environment, and loss of tumor suppressor gene expression can alter oncogene-independent tumor maintenance and recurrence. Oncogene-independent maintenance of c-mycinduced mammary adenocarcinomas is facilitated by ras mutation, the oncogene-independent maintenance of activated Neu-induced adenocarcinomas and metastases is altered when they are transplanted to a different environment, and p53 heterozygosity promotes oncogene-independent maintenance and recurrence of Wnt1-induced adenocarcinomas.

Further exploitation of conditional systems will provide even more detailed information on the diverse molecular mechanisms that act together to produce human cancer. It may be possible to explore earlier stages in the development of cancers using these conditional systems to determine whether premalignant lesions are present, and if so how they regress after oncogene downregulation. In addition to oncogene dependence, the development of the MTB mice, which allow direct targeting of the mouse mammary epithelial cells, allows for the study of other factors that contribute to mammary adenocarcinoma progression. For example, reproductive history is an important risk factor for human breast cancer. It has also been shown in mice that the number of pregnancies hastens the development of mammary adenocarcinoma in HER-2/neu mice [22]. The effect of multiple pregnancies and ovariectomy on tumor latency and cancer regression can also be examined in these models. Use of this knowledge should help us to understand the limitations of current therapies that target specific pathways and to promote development of new ones.

\section{Competing interests}

None declared.

\section{References}

1. Nowell PC: The clonal evolution of tumor cell populations. Science 1976, 194:23-28.

2. Bishop JM: Molecular themes in oncogenesis. Cell 1991, 64: 235-248. 
3. Pitot HC, Dragan YP: Facts and theories concerning the mechanisms of carcinogenesis. FASEB J 1991, 5:2280-2286.

4. Hanahan D, Weinberg RA: The hallmarks of cancer. Cell 2000, 100:57-70.

5. Trosko JE: Commentary: is the concept of 'tumor promotion' a useful paradigm? Mol Carcinog 2001, 30:131-137.

6. Furth PA, St Onge L, Boger H, Gruss P, Gossen M, Kistner A, Bujard $\mathrm{H}$, Hennighausen $\mathrm{L}$ : Temporal control of gene expression in transgenic mice by a tetracycline-responsive promoter. Proc Natl Acad Sci USA 1994, 91:9302-9306.

7. Gossen M, Bujard H: Efficacy of tetracycline-controlled gene expression is influenced by cell type: commentary. Biotechniques 1995, 19:213-216.

8. Kistner A Gossen M, Zimmermann F Jerecic J, Ullmer C Lubbert $H$, Bujard $H$ : Doxycycline-mediated quantitative and tissuespecific control of gene expression in transgenic mice. Proc Natl Acad Sci USA 1996, 93:10933-10938.

9. Gunther EJ, Belka GK, Wertheim GB, Wang J, Hartman JL, Boxer RB, Chodosh LA: A novel doxycycline-inducible system for the transgenic analysis of mammary gland biology. FASEB J 2002, 16:283-292.

10. Efrat $\mathrm{S}$, Fusco-DeMane $\mathrm{D}$, Lemberg $\mathrm{H}$, al Emran $\mathrm{O}$, Wang $\mathrm{X}$ : Conditional transformation of a pancreatic beta-cell line derived from transgenic mice expressing a tetracycline-regulated oncogene. Proc Natl Acad Sci USA 1995, 92:3576-3580.

11. Ewald D, Li M, Efrat $S$, Auer G, Wall RJ, Furth PA, Hennighausen $\mathrm{L}$ : Time-sensitive reversal of hyperplasia in transgenic mice expressing SV40 T antigen. Science 1996, 273:1384-1386.

12. Chin L, Tam A, Pomerantz J, Wong M, Holash J, Bardeesy N, Shen Q, O'Hagan R, Pantginis J, Zhou H, Horner JW, Cordon-Cardo C, Yancopoulos GD, DePinho RA: Essential role for oncogenic Ras in tumour maintenance. Nature 1999, 400:468-472.

13. Fisher GH, Wellen SL, Klimstra D, Lenczowski JM, Tichelaar JW, Lizak MJ, Whitsett JA, Koretsky A, Varmus HE: Induction and apoptotic regression of lung adenocarcinomas by regulation of a K-Ras transgene in the presence and absence of tumor suppressor genes. Genes Dev 2001, 15:3249-3262.

14. Huettner CS, Zhang P, Van Etten RA, Tenen DG: Reversibility of acute B-cell leukaemia induced by BCR-ABL1. Nat Genet 2000, 24:57-60.

15. D'Cruz CM, Gunther EJ, Boxer RB, Hartman JL, Sintasath L, Moody SE, Cox JD, Ha SI, Belka GK, Golant A, Cardiff RD, Chodosh LA: c-MYC induces mammary tumorigenesis by means of a preferred pathway involving spontaneous Kras2 mutations. Nat Med 2001, 7:235-239.

16. Felsher DW, Bishop JM: Reversible tumorigenesis by MYC in hematopoietic lineages. Mol Cell 1999, 4:199-207.

17. Jain M, Arvanitis C, Chu K, Dewey W, Leonhardt E, Trinh M, Sundberg CD, Bishop JM, Felsher DW: Sustained loss of a neoplastic phenotype by brief inactivation of MYC. Science 2002, 297: 102-104.

18. Pelengaris S, Khan M, Evan Gl: Suppression of Myc-induced apoptosis in beta cells exposes multiple oncogenic properties of Myc and triggers carcinogenic progression. Cell 2002, 109:321-334.

19. Moody SE, Sarkisian CJ, Hahn KT, Gunther EJ, Pickup S, Dugan KD, Innocent N, Cardiff RD, Schnall MD, Chodosh LA: Conditional activation of $\mathrm{Neu}$ in the mammary epithelium of transgenic mice results in reversible pulmonary metastasis. Cancer Cell 2002, 2:451-461.

20. Sternlicht MD, Bissell MJ, Werb Z: The matrix metalloproteinase stromelysin-1 acts as a natural mammary tumor promoter. Oncogene 2000, 19:1102-1113.

21. Gunther EJ, Moody SE, Belka GK, Hahn KT, Innocent N, Dugan $\mathrm{KD}$, Cardiff RD, Chodosh LA: Impact of p53 loss on reversal and recurrence of conditional Wnt-induced tumorigenesis. Genes Dev 2003, 17:488-501.

22. Anisimov VN, Popovich IG, Alimova IN, Zabezhinski MA, Semenchenko AV, Yashin Al: Number of pregnancies and ovariectomy modify mammary carcinoma development in transgenic HER-2/neu female mice. Cancer Lett 2003, 193:49-55.

\section{Correspondence}

Priscilla A Furth, Lombardi Cancer Center, Georgetown University, Research Building, Room E518, 3970 Reservoir Road NW, Washington, DC 20057, USA. Tel: +1 202687 8986; fax:+1 202687 7505; e-mail:paf3@georgetown.edu 